\title{
The splittable flow arc set with capacity and minimum load constraints
}

\author{
Hande Yaman* \\ Bilkent University, Department of Industrial Engineering, Bilkent 06800 Ankara, Turkey
}

\section{A R T I C L E I N F O}

\section{Article history:}

Received 27 November 2012

Received in revised form

17 July 2013

Accepted 17 July 2013

Available online 1 August 2013

\section{Keywords:}

Splittable flow arc set

Mixed integer knapsack set

Minimum load constraints

Residual capacity inequalities

Convex hull

\begin{abstract}
A B S T R A C T
We study the convex hull of the splittable flow arc set with capacity and minimum load constraints. This set arises as a relaxation of problems where clients have demand for a resource that can be installed in integer amounts and that has capacity limitations and lower bounds on utilization. We prove that the convex hull of this set is the intersection of the convex hull of the set with a capacity constraint and the convex hull of the set with a minimum load constraint.
\end{abstract}

(c) 2013 Elsevier B.V. All rights reserved.

\section{Introduction}

Let $N=\{1, \ldots, n\}$ be a finite set and suppose that a positive weight $a_{i}$ is associated with each element $i \in N$. Let $u_{0}$ be a nonnegative number and $u$ be a positive number. The single-facility splittable flow arc set is the set of solutions $(x, y) \in \mathbb{R}_{+}^{n} \times \mathbb{Z}_{+}$that satisfy the constraints $\sum_{i \in N} x_{i} \leq u_{0}+u y$ and $x_{i} \leq a_{i}$ for all $i \in N$. This set arises as a relaxation of problems like the capacitated facility location problem [1] and the network loading problem [2], where client $i \in N$ has demand $a_{i}$ for a resource whose existing capacity is $u_{0}$ and for which additional capacity can be installed in integer multiples of $u$. The mixed integer knapsack set with a single integer variable is a generalization of the single-facility splittable flow arc set. It is the set of solutions $(x, y) \in \mathbb{R}_{+}^{n} \times \mathbb{Z}_{+}$that satisfy the constraints $\sum_{i \in N^{+}} x_{i}-\sum_{i \in N^{-}} x_{i} \leq u_{0}+u y$ and $x_{i} \leq a_{i}$ for all $i \in N^{+} \cup N^{-}$where some of $a_{i}$ values may not be finite. The inequalities describing the convex hulls of both sets are known (see Magnanti et al. [2], Atamtürk and Rajan [3], Atamtürk and Günlük [4] and Atamtürk [5]).

In some applications that involve making decisions about capacitated resources, one may also have lower bounds for the load on the resources. This may be due to the fact that installing or setting up a resource may be cost efficient only if a certain load is assigned to it or the system may perform better if the loads

\footnotetext{
* Tel.: +90 31229027 68; fax: +90 3122664054 .

E-mail address: hyaman@bilkent.edu.tr.
}

on the resources are balanced. Some examples are the following. Lim et al. [6] study a transportation problem with minimum quantity commitment imposed by regulations. Constantino [7] studies several relaxations of the lot-sizing problem with lower bounds and Hwang [8] studies a dynamic lot-sizing problem where replenishments cannot be less than a minimum size. Çınar and Yaman [9] study the vendor location problem where each vendor should serve a minimum amount of demand to be profitable. This is an example of the lower bounded (or load balanced) facility location problem [10,11], which generalizes the well-known uncapacitated facility location problem by requiring each open facility to serve no less than a certain amount of demand. Güneş and Yaman [12] study the hospital re-planning problem where a minimum number of patients should be served by each specialty service at an hospital. Galvão et al. [13] consider load balancing as an objective in their hierarchical model applied to the location of perinatal facilities in the municipality of Rio de Janeiro. Güneş et al. [14] use minimum load constraints to improve physician satisfaction in their primary care facility location problem.

Let $\ell_{0}$ be a nonnegative number and $\ell$ be a positive number. The minimum load constraints can be modeled as $\sum_{i \in N} x_{i} \geq \ell_{0}+\ell y$. In this paper, we first study the convex hull of the intersection of two mixed integer sets with a single integer variable in a special case and show that the convex hull of the intersection is the intersection of the convex hulls. Then we apply this result to the mixed integer knapsack set with a capacity and minimum load constraint. Using the description of the convex hull of the mixed integer knapsack set and properties of extreme points, we also give the description 
for the splittable flow arc set with two integer variables when one of the capacities is a unit capacity and all parameters are integers.

The rest of the paper is organized as follows. In Section 2, we give the theorem about the convex hull of the intersection of two mixed integer sets with a single integer variable. In Section 3, the result of the previous section is used to describe the convex hulls of the mixed integer knapsack set and the splittable flow arc set with both capacity and minimum load constraints. Conclusions are given in Section 4.

\section{Intersection of two mixed integer sets with a single integer variable}

In the sequel, we assume all data to be rational. Let $g \in \mathbb{R}^{1 \times n}$, $\bar{g}_{1}, \bar{g}_{2}, b_{1}, b_{2} \in \mathbb{R}, \hat{G} \in \mathbb{R}^{q \times n}$, and $\hat{g}, \hat{b} \in \mathbb{R}^{q}$, and consider the sets

$P^{1}=\left\{(x, y) \in \mathbb{R}^{n+1}: g x+\bar{g}_{1} y \leq b_{1}, \hat{G} x+\hat{g} y \leq \hat{b}\right\}$,

$P^{2}=\left\{(x, y) \in \mathbb{R}^{n+1}:-g x+\bar{g}_{2} y \leq b_{2}, \hat{G} x+\hat{g} y \leq \hat{b}\right\}$,

$X^{1}=P^{1} \cap\left(\mathbb{R}^{n} \times \mathbb{Z}\right), X^{2}=P^{2} \cap\left(\mathbb{R}^{n} \times \mathbb{Z}\right)$, and their intersections $P=P^{1} \cap P^{2}$ and $X=X^{1} \cap X^{2}$, where the system $\hat{G} x+\hat{g} y \leq \hat{b}$ includes $y \geq 0$.

Theorem 1. If $\left(\bar{g}_{1}+\bar{g}_{2}\right)\left(b_{1}+b_{2}\right) \leq 0$, then $\operatorname{conv}(X)=\operatorname{conv}\left(X^{1}\right) \cap$ $\operatorname{conv}\left(X^{2}\right)$.

Proof. We use the fact that the split cuts are sufficient to describe the convex hull of a mixed integer set with a single integer variable (see $[15,16])$. Let $d$ be a nonnegative integer and consider the disjunction $y \leq d$ and $y \geq d+1$ and the associated split cut $\beta x+\alpha y \leq$ $\delta$ for $P$. Following the treatment of Di Summa [16], by the Farkas Lemma, there exist multipliers $v_{1}, v_{2}, \hat{v}, w_{1}, w_{2}, \hat{w}, \lambda, \mu \geq 0$ such that $v_{1} g-v_{2} g+\hat{v} \hat{G}=w_{1} g-w_{2} g+\hat{w} \hat{G}=\beta, v_{1} \bar{g}_{1}+v_{2} \bar{g}_{2}+\hat{v} \hat{g}+\lambda=$ $w_{1} \bar{g}_{1}+w_{2} \bar{g}_{2}+\hat{w} \hat{g}-\mu=\alpha$, and $v_{1} b_{1}+v_{2} b_{2}+\hat{v} \hat{b}+\lambda d=w_{1} b_{1}+$ $w_{2} b_{2}+\hat{w} \hat{b}-\mu(d+1)=\delta$. Solving the last two equations, we obtain $\mu=\left(w_{1}-v_{1}\right)\left(b_{1}-d \bar{g}_{1}\right)+\left(w_{2}-v_{2}\right)\left(b_{2}-d \bar{g}_{2}\right)+(\hat{w}-\hat{v})(\hat{b}-d \hat{g})$ and $\lambda=\left(v_{1}-w_{1}\right)\left(b_{1}-(d+1) \bar{g}_{1}\right)+\left(v_{2}-w_{2}\right)\left(b_{2}-(d+1) \bar{g}_{2}\right)+$ $(\hat{v}-\hat{w})(\hat{b}-(d+1) \hat{g})$.

Let $\mathcal{C}$ be the cone of nonnegative vectors $\left(v_{1}, v_{2}, \hat{v}, w_{1}, w_{2}, \hat{w}\right.$, $\lambda, \mu)$ that satisfy

$$
\begin{aligned}
\left(v_{1}-\right. & \left.w_{1}\right) g-\left(v_{2}-w_{2}\right) g+(\hat{v}-\hat{w}) \hat{G}=0, \\
\lambda= & \left(v_{1}-w_{1}\right)\left(b_{1}-(d+1) \bar{g}_{1}\right)+\left(v_{2}-w_{2}\right)\left(b_{2}-(d+1) \bar{g}_{2}\right) \\
& +(\hat{v}-\hat{w})(\hat{b}-(d+1) \hat{g}), \\
\mu= & \left(w_{1}-v_{1}\right)\left(b_{1}-d \bar{g}_{1}\right)+\left(w_{2}-v_{2}\right)\left(b_{2}-d \bar{g}_{2}\right) \\
& +(\hat{w}-\hat{v})(\hat{b}-d \hat{g}) .
\end{aligned}
$$

A nondominated split cut for $P$ is given by a nonnegative integer $d$ and an extreme ray of the cone $\mathcal{C}$ for which $\mu>0$ and $\lambda>0$ (if $\mu=0$ or $\lambda=0$ then the split cut is implied by the original system [16]).

Suppose that $p=\left(v_{1}, v_{2}, \hat{v}, w_{1}, w_{2}, \hat{w}, \lambda, \mu\right)$ is an extreme ray of $\mathcal{C}$ with $v_{1}>0, v_{2}>0, \lambda>0$ and $\mu>0$. Let $\epsilon>0$ be a very small number and consider the vectors $p^{1}=\left(v_{1}+\epsilon, v_{2}+\epsilon, \hat{v}\right.$, $\left.w_{1}, w_{2}, \hat{w}, \lambda^{1}, \mu^{1}\right)$ and $p^{2}=\left(v_{1}-\epsilon, v_{2}-\epsilon, \hat{v}, w_{1}, w_{2}, \hat{w}, \lambda^{2}, \mu^{2}\right)$ where $\lambda^{1}, \mu^{1}, \lambda^{2}$ and $\mu^{2}$ are computed using the last two equalities and they remain nonnegative as $\epsilon$ is very small. These vectors $p^{1}$ and $p^{2}$ are in $\mathcal{C}$ and $p=1 / 2 p^{1}+1 / 2 p^{2}$. As $p$ is an extreme ray, $p^{1}$ and $p^{2}$ should be multiples of $p$. This implies that $\hat{v}=w_{1}=w_{2}=$ $\hat{w}=0$, and $v_{1}=v_{2}$. Then $\lambda=v_{1}\left(b_{1}+b_{2}-(d+1)\left(\bar{g}_{1}+\bar{g}_{2}\right)\right)$ and $\mu=v_{1}\left(-\left(b_{1}+b_{2}\right)+d\left(\bar{g}_{1}+\bar{g}_{2}\right)\right)$. For $\lambda$ and $\mu$ to be positive, we need $b_{1}+b_{2}>(d+1)\left(\bar{g}_{1}+\bar{g}_{2}\right)$ and $d\left(\bar{g}_{1}+\bar{g}_{2}\right)>b_{1}+b_{2}$. As $d$ is nonnegative, this implies that $\bar{g}_{1}+\bar{g}_{2}<0$ and $b_{1}+b_{2}<0$. Now, since $\left(\bar{g}_{1}+\bar{g}_{2}\right)\left(b_{1}+b_{2}\right) \leq 0$, at least one of $\lambda$ and $\mu$ is not positive. Hence any split cut derived from an extreme ray with $v_{1}>0$ and $v_{2}>0$ is dominated.
Similarly, one can show that if $p$ with $w_{1}>0$ and $w_{2}>0$ is an extreme ray of $\mathcal{C}$, then $w_{1}=w_{2}$ and $v_{1}=v_{2}=\hat{v}=\hat{w}=0$. In this case, $\lambda=w_{1}\left(-b_{1}+(d+1) \bar{g}_{1}-b_{2}+(d+1) \bar{g}_{2}\right)$ and $\mu=$ $w_{1}\left(b_{1}-d \bar{g}_{1}+b_{2}-d \bar{g}_{2}\right)$. We need $\bar{g}_{1}+\bar{g}_{2}>0$ and $b_{1}+b_{2}>0$ for $\lambda$ and $\mu$ to be positive. This is not possible since $\left(\bar{g}_{1}+\bar{g}_{2}\right)\left(b_{1}+b_{2}\right) \leq 0$.

Let $p \in \mathcal{C}$ with $v_{1}>0$ and $w_{2}>0$. Both $p^{1}=\left(v_{1}+\epsilon, v_{2}, \hat{v}, w_{1}\right.$, $\left.w_{2}-\epsilon, \hat{w}, \lambda^{1}, \mu^{1}\right)$ and $p^{2}=\left(v_{1}-\epsilon, v_{2}, \hat{v}, w_{1}, w_{2}+\epsilon, \hat{w}, \lambda^{2}, \mu^{2}\right)$, where $\lambda^{1}, \mu^{1}, \lambda^{2}$ and $\mu^{2}$ are computed using the last two equalities, are in $C$ and $p=1 / 2 p^{1}+1 / 2 p^{2}$. Since $p_{1}$ and $p_{2}$ cannot be multiples of $p, p$ is not an extreme ray. The case with $v_{2}>0$ and $w_{1}>0$ is similar.

Hence, we can conclude that nondominated split cuts are generated by extreme rays $p$ where either $v_{1}=w_{1}=0$ or $v_{2}=$ $w_{2}=0$. A ray $p$ with $v_{2}=w_{2}=0, \lambda>0$ and $\mu>0$ is an extreme ray of $\mathcal{C}$ if and only if $\left(v_{1}, \hat{v}, w_{1}, \hat{w}, \lambda, \mu\right)$ is an extreme ray of $\mathcal{C}_{1}=\left\{\left(v_{1}, \hat{v}, w_{1}, \hat{w}, \lambda, \mu\right) \geq 0:\left(v_{1}-w_{1}\right) g+(\hat{v}-\hat{w}) \hat{G}=0, \mu=\right.$ $\left(w_{1}-v_{1}\right)\left(b_{1}-d \bar{g}_{1}\right)+(\hat{w}-\hat{v})(\hat{b}-d \hat{g}), \lambda=\left(v_{1}-w_{1}\right)\left(b_{1}-(d+\right.$ 1) $\left.\left.\bar{g}_{1}\right)+(\hat{v}-\hat{w})(\hat{b}-(d+1) \hat{g})\right\}$. Similarly, $p$ with $v_{1}=w_{1}=0, \lambda>0$ and $\mu>0$ is an extreme ray of $\mathcal{C}$ if and only if $\left(v_{2}, \hat{v}, w_{2}, \hat{w}, \lambda, \mu\right)$ is an extreme ray of $\mathcal{C}_{2}=\left\{\left(v_{2}, \hat{v}, w_{2}, \hat{w}, \lambda, \mu\right) \geq 0:-\left(v_{2}-\right.\right.$ $\left.w_{2}\right) g+(\hat{v}-\hat{w}) \hat{G}=0, \mu=\left(w_{2}-v_{2}\right)\left(b_{2}-d \bar{g}_{2}\right)+(\hat{w}-\hat{v})(\hat{b}-$ $\left.d \hat{g}), \lambda=\left(v_{2}-w_{2}\right)\left(b_{2}-(d+1) \bar{g}_{2}\right)+(\hat{v}-\hat{w})(\hat{b}-(d+1) \hat{g})\right\}$.

Hence, we can conclude that an inequality is a nondominated split cut for $P$ if and only if it is a nondominated split cut for $P^{1}$ or for $P^{2}$.

\section{Applications}

\subsection{The mixed integer knapsack set with a single integer variable}

Suppose that we are given two sets of items $N^{+}$and $N^{-}$with $\left|N^{+}\right|+\left|N^{-}\right|=n$, a positive parameter $a_{i}$ for each item $i \in N^{+} \cup N^{-}$ (possibly infinite) and two finite numbers $u_{0}$ and $u$. The mixed integer knapsack set with a single integer variable is the set of solutions $(x, y) \in \mathbb{R}_{+}^{n} \times \mathbb{Z}_{+}$that satisfy the constraints $\sum_{i \in N^{+}} x_{i}-\sum_{i \in N^{-}} x_{i} \leq$ $u_{0}+u y$ and $x_{i} \leq a_{i}$ for all $i \in N^{+} \cup N^{-}$. This set is a generalization of the single-facility splittable flow arc set with a capacity constraint.

Atamtürk [5] presents facet defining inequalities for the polyhedron of the mixed integer knapsack set. He first gives the description of the convex hull of the set with a single integer variable and then applies sequential lifting.

Here, using the results of Atamtürk [5] and the theorem of the previous section, we give the description of the convex hull of a mixed integer knapsack set with capacity and load constraints. This is the set of solutions to

$\sum_{i \in N^{+}} x_{i}-\sum_{i \in N^{-}} x_{i} \leq u_{0}+u y$,
$\sum_{i \in N^{+}} x_{i}-\sum_{i \in N^{-}} x_{i} \geq \ell_{0}+\ell y$,

$0 \leq x_{i} \leq a_{i} \quad i \in N^{+} \cup N^{-}$,

$y \in \mathbb{Z}_{+}$.

We assume that $u \geq \ell$ and $u_{0} \geq \ell_{0}$.

Let $X_{C}=\left\{(x, y) \in \mathbb{R}^{n+1}:(1),(3),(4)\right\}, X_{L}=\left\{(x, y) \in \mathbb{R}^{n+1}\right.$ : (2)-(4) $\}$ and $X_{L C}=\left\{(x, y) \in \mathbb{R}^{n+1}:(1)-(4)\right\}=X_{C} \cap X_{L}$.

Let $B=\left\{i \in N^{+} \cup N^{-}: a_{i}\right.$ is finite $\}$. For $S \subseteq N^{+} \cup N^{-}$, let $a(S)=\sum_{i \in S} a_{i}$.

Atamtürk [5] gives the descriptions of $\operatorname{conv}\left(X_{C}\right)$ and $\operatorname{conv}\left(X_{L}\right)$. $\operatorname{conv}\left(X_{C}\right)$ is described by the original constraints and the inequalities

$\sum_{i \in N^{+} \cap S}\left(a_{i}-x_{i}\right)+\sum_{i \in N^{-} \cap S} x_{i}+\sum_{i \in N^{-} \backslash B} x_{i} \geq r_{S}^{+}\left(\eta_{S}^{+}-y\right)$ 
for all $S \subseteq B$, where $\eta_{S}^{+}=\left\lceil\frac{a(S)-u_{0}-a\left(N^{-} \cap B\right)}{u}\right\rceil$ and $r_{S}^{+}=a(S)-u_{0}-$ $a\left(N^{-} \cap B\right)-\left|\frac{a(S)-u_{0}-a\left(N^{-} \cap B\right)}{u}\right| u>0$.

$\operatorname{conv}\left(X_{L}\right)$ is described by the original constraints and the inequalities

$\sum_{i \in N^{+} \cap T} x_{i}-\sum_{i \in N^{-} \cap T} x_{i}+\sum_{i \in N^{+} \backslash B} x_{i} \geq r_{T}^{-}\left(y-\eta_{T}^{-}\right)-a\left(N^{-} \cap T\right)$

for all $T \subseteq B$, where $\eta_{T}^{-}=\left\lfloor\frac{a\left(N^{+} \cap B\right)-\ell_{0}-a(T)}{\ell}\right\rfloor$ and $r_{T}^{-}=$ $\left\lceil\frac{a\left(N^{+} \cap B\right)-\ell_{0}-a(T)}{\ell}\right\rceil \ell-a\left(N^{+} \cap B\right)+\ell_{0}+a(T)>0$.

Corollary 1. $\operatorname{conv}\left(X_{L C}\right)$ is described by constraints (1)-(3), the nonnegativity constraint for $y$, inequalities (5) for all $S \subseteq B$ with $r_{S}^{+}>0$ and inequalities (6) for all $T \subseteq B$ with $r_{T}^{-}>0$.

\subsection{The two-facility splittable flow arc set}

Magnanti et al. [2] study the single-facility splittable flow arc set with a capacity constraint and derive a family of valid inequalities called the "residual capacity inequalities". They prove that these inequalities together with the original constraints describe the convex hull when $u_{0}=0$. Atamtürk and Rajan [3] state without proof that these results generalize to the case of arbitrary $u_{0}$ and present a polynomial time separation algorithm for the residual capacity inequalities. The convex hull proof for arbitrary $u_{0}$ is given by Atamtürk and Günlük [4]. Later, Magnanti et al. [17] state without proof that the constraints and the residual capacity inequalities describe the convex hull of the two-facility splittable flow arc set with a capacity constraint where the resource can be installed in units or in multiples of $u$ in the special case where $u_{0}=0$, and $a_{i}$ for $i \in N$ and $u$ are integers.

Here, we study the two-facility splittable flow arc set with a capacity and minimum load constraint. Consider the mixed integer knapsack set with $\left|N^{-}\right|=1, N^{-} \cap B=\emptyset$ and $N=N^{+}=B$. In this case, we obtain the set described by the system

$\sum_{i \in N} x_{i} \leq u_{0}+y_{0}+u y$

$\sum_{i \in N} x_{i} \geq \ell_{0}+y_{0}+\ell y$,

$0 \leq x_{i} \leq a_{i} \quad i \in N$,

$y_{0} \geq 0$,

$y \in \mathbb{Z}_{+}$,

where $y_{0}$ is the variable whose index is in $N^{-}$. We assume that all parameters are integers. We are interested in the convex hull of the set $Y_{L C}=X_{L C} \cap\left\{\left(x, y_{0}, y\right): y_{0}\right.$ integer $\}$.

First, note that, in this special case, inequality (5) becomes

$\sum_{i \in S}\left(a_{i}-x_{i}\right)+y_{0} \geq r_{S}^{+}\left(\eta_{S}^{+}-y\right)$

for $S \subseteq N, \eta_{S}^{+}=\left\lceil\frac{a(S)-u_{0}}{u}\right\rceil$ and $r_{S}^{+}=a(S)-u_{0}-\left\lfloor\frac{a(S)-u_{0}}{u}\right\rfloor u$. This inequality is the residual capacity inequality given in Magnanti et al. [17] (with $u_{0}=0$ ). Similarly, inequality (6) becomes

$\sum_{i \in T} x_{i} \geq r_{T}^{-}\left(y-\eta_{T}^{-}\right)$

for $T \subseteq N, r_{T}^{-}=\left\lceil\frac{a(N \backslash T)-\ell_{0}}{\ell}\right\rceil \ell-a(N \backslash T)+\ell_{0}$ and $\eta_{T}^{-}=$ $\left\lfloor\frac{a(N \backslash T)-\ell_{0}}{\ell}\right\rfloor$. We refer to this inequality as the "residual load inequality".
Theorem 2. If $a_{i}$ for $i \in N, u_{0}, u, \ell_{0}$, and $\ell$ are integers, then conv $\left(Y_{L C}\right)$ is described by constraints (7)-(9), the nonnegativity constraints for $y_{0}$ and $y$, inequalities (11) for all $S \subseteq N$ with $r_{S}^{+}>0$ and inequalities (12) for all $T \subseteq N$ with $r_{T}^{-}>0$.

Proof. By Corollary 1, we know that the constraints and the residual capacity and residual load inequalities are sufficient to describe $\operatorname{conv}\left(X_{L C}\right)$. Let $\left(x, y_{0}, y\right) \in \operatorname{conv}\left(X_{L C}\right)$ with $y \in \mathbb{Z}$ and $y_{0} \notin \mathbb{Z}$ and define $N^{\prime}=\left\{i \in N: 0<x_{i}<a_{i}\right\}$. If $N^{\prime}=\emptyset$, then as all parameters are integers, both $\left(x, y_{0}-\epsilon, y\right)$ and $\left(x, y_{0}+\epsilon, y\right)$ are also in $X_{L C}$ for very small $\epsilon>0$ and $\left(x, y_{0}, y\right)$ is not an extreme point of $\operatorname{conv}\left(X_{L C}\right)$. Now suppose that $N^{\prime} \neq \emptyset$. Let $j \in N^{\prime}$ and consider the points $\left(x^{1}, y_{0}^{1}, y\right)$ and $\left(x^{2}, y_{0}^{2}, y\right)$, where $x_{j}^{1}=x_{j}-\epsilon, x_{j}^{2}=x_{j}+\epsilon, y_{0}^{1}=y_{0}-\epsilon$, $y_{0}^{2}=y_{0}+\epsilon$, and $x_{i}^{1}=x_{i}^{2}=x_{i}$ for $i \in N \backslash\{j\}$. The points $\left(x^{1}, y_{0}^{1}, y\right)$ and $\left(x^{2}, y_{0}^{2}, y\right)$ satisfy all the constraints and they are in $X_{L C}$. This proves that $\left(x, y_{0}, y\right)$ is not an extreme point of $\operatorname{conv}\left(X_{L C}\right)$. Hence all extreme points of $\operatorname{conv}\left(X_{L C}\right)$ have integral $y$ and $y_{0}$ values.

\section{Conclusion}

An interesting question that remains for further studies is how one can describe the convex hulls of the two-facility splittable flow arc sets when some of the parameters are fractional. Related to this, we also would like to investigate whether the intersection result holds true for the splittable flow arc sets with more than two integer variables.

\section{Acknowledgments}

The author is grateful to the associate editor for pointing out a mistake in one of the proofs. The research of the author was supported by the Turkish Academy of Sciences.

\section{References}

[1] J.M.Y. Leung, T.L. Magnanti, Valid inequalities and facets of the capacitated plant location problem, Math. Program. 44 (1989) 271-291.

[2] T.L. Magnanti, P. Mirchandani, R. Vachani, The convex hull of two core capacitated network design polyhedra, Math. Program. 60 (1993) 233-250.

[3] A. Atamtürk, D. Rajan, On splittable and unsplittable flow capacitated network design arc-set polyhedra, Math. Program. 92 (2002) 315-333.

[4] A. Atamtürk, O. Günlük, Network design arc set with variable upper bounds, Networks 50 (2007) 17-28.

[5] A. Atamtürk, On the facets of the mixed-integer knapsack polyhedron, Mathematical Programming B 98 (2003) 145-175.

[6] A. Lim, F. Wang, Z. Xu, A transportation problem with minimum quantity commitment, Transp. Sci. 40 (2006) 117-129.

[7] M. Constantino, Lower bounds in lot-sizing models: a polyhedral study, Math. Oper. Res. 23 (1998) 101-118.

[8] H.C. Hwang, Inventory replenishment and inbound shipment scheduling under a minimum replenishment policy, Transp. Sci. 43 (2009) 244-264.

[9] Y. Çınar, H. Yaman, The vendor location problem, Comput. Oper. Res. 38 (2011) 1678-1695.

[10] S. Guha, A. Meyerson, K. Munagala, Hierarchical placement and network design problems, in: Proc. 41st IEEE Symp. on Foundations of Computer Science, 2000, pp. 603-612.

[11] D.R. Karger, M. Minkoff, Building Steiner trees with incomplete global knowledge, in: Proc. 41st IEEE Symp. on Foundations of Computer Science, 2000, pp. 613-623.

[12] E.D. Güneş, H. Yaman, Health network mergers and hospital re-planning, J. Oper. Res. Soc. 61 (2010) 275-283.

[13] R.D. Galvão, L.G.A. Espejo, B. Boffey, D. Yates, Load balancing and capacity constraints in a hierarchical location model, European J. Oper. Res. 172 (2006) 631-646.

[14] E.D. Güneş, H. Yaman, B. Çekyay, V. Verter, Matching patient and physician preferences in designing a primary care facility network, J. Oper. Res. Soc., in press, http://dx.doi.org/10.1057/jors.2012.71.

[15] W. Cook, R. Kannan, A. Schrijver, Chvatal closures for mixed integer programming problems, Math. Program. 47 (1990) 155-174.

[16] M. Di Summa, On a class of mixed-integer sets with a single integer variable, Oper. Res. Lett. 38 (2010) 556-558.

[17] T.L. Magnanti, P. Mirchandani, R. Vachani, Modeling and solving the twofacility capacitated network loading problem, Oper. Res. 43 (1995) 142-157. 